\title{
Pseudoneglect in Visual Search: Behavioral Evidence and Connectional Constraints in Simulated Neural Circuitry
}

\author{
(1) Onofrio Gigliotta, ${ }^{1}$ (1)Tal Seidel Malkinson, ${ }^{2}$ Orazio Miglino, ${ }^{1,3}$ and ${ }^{-P a o l o}$ Bartolomeo ${ }^{2}$
}

\section{DOI:http://dx.doi.org/10.1523/ENEURO.0154-17.2017}

\begin{abstract}
${ }^{1}$ Department of Humanistic Studies, University of Naples Federico II, 80133 Naples, Italy, ${ }^{2}$ Institut National de la Santé et de la Recherche Médicale Unité 1127, Centre National de la Recherche Scientifique Unité Mixte de Recherche (UMR) 7225, Sorbonne Universités, Université Pierre-et-Marie-Curie Univ Paris 06 UMR S 1127, Institut du Cerveau et de la Moelle Épinière ICM, 75013 Paris, France, and ${ }^{3}$ Institute of Cognitive Sciences and Technologies, National Research Council, 00185 Rome, Italy
\end{abstract}

\begin{abstract}
Most people tend to bisect horizontal lines slightly to the left of their true center (pseudoneglect) and start visual search from left-sided items. This physiological leftward spatial bias may depend on hemispheric asymmetries in the organization of attentional networks, but the precise mechanisms are unknown. Here, we modeled relevant aspects of the ventral and dorsal attentional networks (VAN and DAN) of the human brain. First, we demonstrated pseudoneglect in visual search in 101 right-handed psychology students. Participants consistently tended to start the task from a left-sided item, thus showing pseudoneglect. Second, we trained populations of simulated neurorobots to perform a similar task, by using a genetic algorithm. The neurorobots' behavior was controlled by artificial neural networks, which simulated the human VAN and DAN in the two brain hemispheres. Neurorobots differed in the connectional constraints that were applied to the anatomy and function of the attention networks. Results indicated that (1) neurorobots provided with a biologically plausible hemispheric asymmetry of the VAN-DAN connections, as well as with interhemispheric inhibition, displayed the best match with human data; however; (2) anatomical asymmetry per se was not sufficient to generate pseudoneglect; in addition, the VAN must have an excitatory influence on the ipsilateral DAN; and (3) neurorobots provided with bilateral competence in the VAN but without interhemispheric inhibition failed to display pseudoneglect. These findings provide a proof of concept of the causal link between connectional asymmetries and pseudoneglect and specify important biological constraints that result in physiological asymmetries of human behavior.
\end{abstract}

Key words: attention; brain connections; spatial exploration; spatial neglect; visual search

\section{Significance Statement}

Most of us start our exploration of the environment from the left side. Here, we demonstrated this tendency in undergraduate students, and trained artificial agents (neurorobots) to perform a similar visual search task. The neurorobots' behavior was controlled by artificial neural networks, inspired by the human fronto-parietal attentional system. In seven distinct populations of neurorobots, different constraints were applied on the network connections within and between the brain hemispheres. Only one of the artificial populations behaved in a similar way to the human participants. The connectional constraints applied to this population included known characteristics of the human fronto-parietal networks but had also additional properties not previously described. Thus, our findings specify biological constraints that induce physiologic asymmetries of human behavior. 


\section{Introduction}

A thorough exploration of the space around us is essential to everyday life. However, spatial exploration is not perfectly symmetrical in humans. For example, when we explore a scene to cancel out visual targets, we tend to start the search from the left part of the scene (Bartolomeo et al., 1994; Azouvi et al., 2006). This physiological leftward spatial bias is analogous to the slight physiological leftward shift typically observed in horizontal line bisection, termed pseudoneglect (Bowers and Heilman, 1980), because it goes in the opposite direction to the typical rightward bias showed by patients with left visual neglect after right hemisphere damage (Schenkenberg et al., 1980; Urbanski and Bartolomeo, 2008).

Evidence shows that visuospatial attention is a major determinant of pseudoneglect (McCourt et al., 2005; Toba et al., 2011), which might thus result from asymmetries in the hemispheric control of attention (McCourt and Jewell, 1999; Ossandón et al., 2014). However, the specific neural structures and the mechanisms at the basis of pseudoneglect remain unknown.

In the human brain, visuospatial attention is controlled by fronto-parietal networks, which demonstrate substantial asymmetries favoring the right hemisphere (Heilman and Van Den Abell, 1980; Mesulam, 1999; Corbetta and Shulman, 2002). Dysfunction of these networks after right hemisphere damage can induce signs of neglect for leftsided events (Corbetta and Shulman, 2011; Bartolomeo et al., 2012). A bilateral dorsal attentional network (DAN), composed by the intraparietal sulcus/superior parietal lobule and the frontal eye field/dorsolateral prefrontal cortex, shows increased BOLD responses during the orienting period (Corbetta and Shulman, 2002). A rightlateralized ventral attentional network (VAN) includes the temporoparietal junction and the ventrolateral prefrontal cortex. The VAN is important for detecting unexpected but behaviorally relevant events, and induces the DANs to reorient attention toward these events. Anatomically, three branches of a long-range white matter pathway, the superior longitudinal fasciculus (SLF), connect these networks. The SLF has a ventro-dorsal gradient of hemispheric asymmetry (Thiebaut de Schotten et al., 2011). The ventral branch (SLF III) connects the VAN and is anatomically larger in the right hemisphere than in the left

Received May 9, 2017; accepted December 4, 2017; First published December 19, 2017

The authors declare no competing financial interests.

Author contributions: O.G., T.S.M., O.M., and P.B. designed research; O.G. performed research; O.G., and T.S.M. analyzed data; O.G., T.S.M., O.M., and P.B. wrote the paper.

This work was supported by the Israeli Science Foundation Grant ISF 57/15 (to T.S.M.), the Program Investissements d'Avenir Grant ANR-10-IAIHU-06, the University Federico II, and ISTC CNR, Rome, Italy.

Correspondence should be addressed to Paolo Bartolomeo, Institut du Cerveau et de la Moelle Epiniere, Hopital Pitie-Salpetriere, 47 Boulevard De L'Hopital, CS 21414, 75646 Paris Cedex 13, E-mail: paolo.bartolomeo@gmail.com.

DOl:http://dx.doi.org/10.1523/ENEURO.0154-17.2017

Copyright (c) 2017 Gigliotta et al.

This is an open-access article distributed under the terms of the Creative Commons Attribution 4.0 International license, which permits unrestricted use, distribution and reproduction in any medium provided that the original work is properly attributed. hemisphere, whereas the dorsal branch (SLF I, connecting the DAN) is more symmetrical. The lateralization of the intermediate branch (SLF II) displays interindividual differences, and is strongly correlated to the individual amount of pseudoneglect in line bisection and to differences in the speed of detection between left-sided and right-sided targets. Specifically, larger SLF volumes in the right hemisphere correlate with larger degrees of leftward bias (Thiebaut de Schotten et al., 2011).

A further potential source of performance asymmetry resides in the pattern of interhemispheric connections. Behavioral and electrophysiological evidence suggests that interhemispheric communication is not strictly symmetrical in humans, but it is faster from the right to the left hemisphere (Marzi, 2010). Also, the posterior callosal connections from the right parietal node of the DAN to its left hemisphere homolog seem to be predominantly inhibitory (Koch et al., 2011). Concerning the VAN, its right and left temporo-parietal caudal nodes are not strongly connected by callosal fibers (Catani and Thiebaut de Schotten, 2012) and, thus, work in relative isolation from one another.

It is tempting to relate these biological constraints to the widespread leftward bias that occurs in human exploratory behavior. However, little is known about the specific dynamic interplay between the attentional networks resulting in pseudoneglect. On the one hand, methods used in humans have substantial limitations of spatiotemporal resolution and of inferential power, which severely limit their scope. On the other hand, it is difficult to draw firm conclusions from monkey neurophysiology, because of important differences between humans and primates in the organization of attention networks (Patel et al., 2015). In the present study, we took a different approach to unravel these issues. First, we tested a group of human participants to establish the presence and characteristics of pseudoneglect in a visual search task (experiment 1). In experiment 2 , we trained neurally controlled robots (neurorobots) to perform a task as similar as possible to the human one. We then articulated detailed implementations of several instances of attention network architecture, which directed the neurorobots' performance, to identify the structural and functional network constraints crucial for simulating human performance.

\section{Experiment 1: Pseudoneglect in Human Visual Search}

\section{Introduction}

Pseudoneglect has been mainly measured using tasks of perceptual estimation of the length of horizontal lines (Bowers and Heilman, 1980; Jewell and McCourt, 2000; Toba et al., 2011). Analogous leftward biases seem also to occur in visual search tasks, as a tendency to find first targets on the left side of the display (Bartolomeo et al., 1994; Azouvi et al., 2006), but evidence in this domain is much less systematic. Thus, in the present context, it was important to test our specific task to ensure the validity of the human-robotic comparison of performance. 


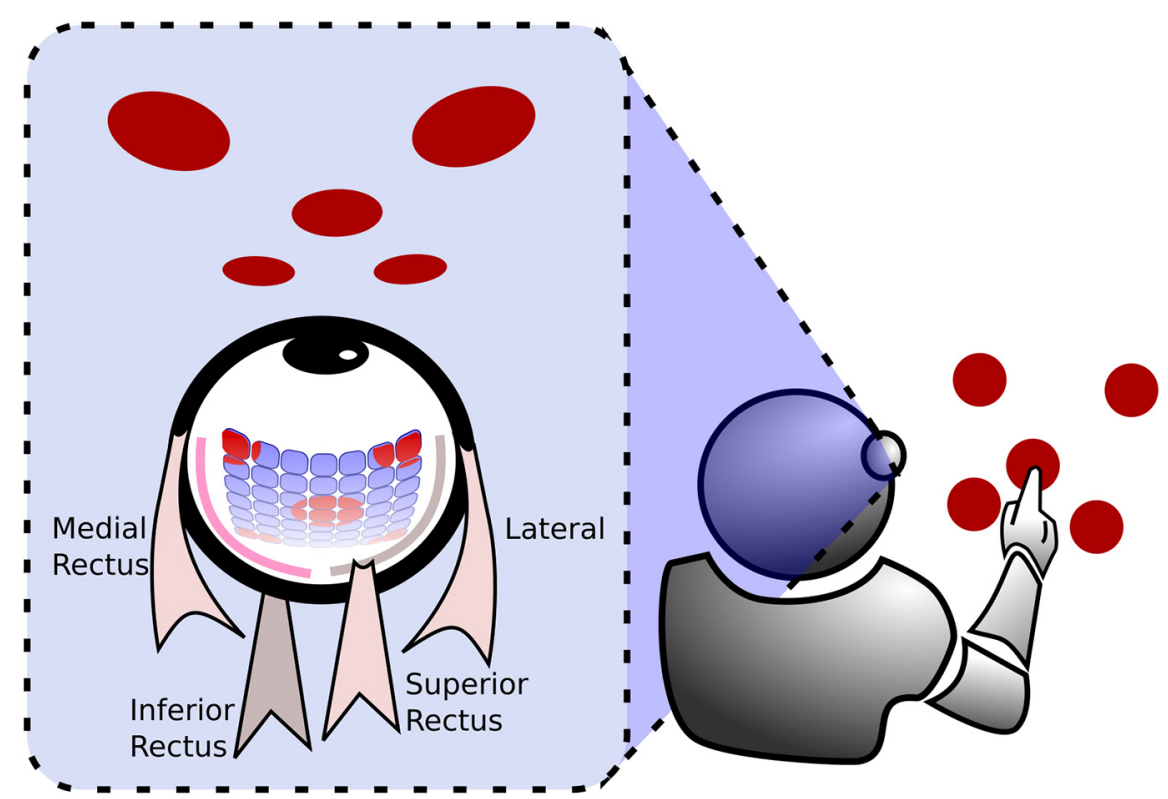

Figure 1. Schema of the neurorobot equipped with an artificial eye, provided with a $7 \times 7$ light receptor retina and controlled by two pairs of simulated extraocular muscles.

\section{Materials and Methods}

Ethics statement

The procedure was approved by the local ethics committee.

\section{Participants}

A total of 101 right-handed psychology students (76 females; mean age \pm SD, $22.24 \pm 4.40$ ) gave their informed consent to perform a visual search experiment for course credit.

\section{Procedure}

Participants were instructed to cancel as fast as possible targets displayed on a touch-sensitive tablet (Mediacom Winpad 8018 -inches, $120 \mathrm{dpi}, 1280 \times 800$ pixels, refresh frequency $60 \mathrm{~Hz}$ ), by using a stylus pen. Participants were comfortably seated with a viewing distance of $\sim 40 \mathrm{~cm}$. Each session consisted of 30 trials. Each trial was initiated by the participant touching a green round button placed at the center of the screen. Subsequently, a set of five dark-red (HEX \#800000) filled round targets, with a 40 -pixel radius $\left(0.76^{\circ}\right.$ visual angle), was presented. Targets were randomly scattered on a display area of 512 $\times 512$ pixels $\left(9.7^{\circ} \times 9.7^{\circ}\right)$, placed at the center of the screen. On participant's touch, cancelled targets became bright red (HEX \#FF0000). To assess lateral bias, we first defined the center of the display as 0 , so that the values of the $X$ coordinate went from -256 pixels $\left(-4.85^{\circ}\right)$ on the extreme left to +256 pixels $\left(+4.85^{\circ}\right)$ on the extreme right. Second, we measured the average position on the $x$-axis of the first cancelled stimulus for each trial.

\section{Results}

As expected with this easy task, accuracy was at ceiling, with all participants correctly cancelling all the targets. Results showed a left-biased distribution of the first found target. The average $X$ value was -80.23 pixels $\left(-1.52^{\circ}\right)$, which significantly differs from the central posi- tion at $X=0$ (Wilcoxon-Mann-Whitney two-tailed test, $Z=-6.37, p<0.001)$.

\section{Discussion}

During a visual search task similar to that used for our simulations, normal participants exhibited a leftward bias (pseudoneglect), consisting of a tendency to start the visual search from a left-sided target. This result was observed in an experimental setting as close as possible to that used for neurorobots, and replicates and extends previous results obtained with different types of visual search tasks, such as the line cancellation test (Bartolomeo et al., 1994) and the bells test (Rousseaux et al., 2001).

\section{Experiment 2: Visual Search in Neurorobots}

\section{Introduction}

A neurorobot is a real or simulated robot whose behavior is controlled by an artificial neural network. For the present experiment, we developed distinct populations of simulated neurorobots controlled by artificial neural networks with different connectivity constraints. The neurorobots' task was designed to be as close as possible to that performed by human participants in experiment 1.

\section{Models}

Code Accessibility: The code is available as Extended Data 1 and in GitHub repository (Gigliotta, 2017).

The simulated robot (Fig. 1) has a single artificial eye and an actuator (simulated hand) able to perform the cancellation task. The robot's eye can move and zoom, and can thus be described as a pan/tilt/zoom camera, because it can move along the horizontal and vertical axes and can zoom in a range between $1 \times$ and $12 \times$. The use of a zoom was inspired by models of attention, which stipulate that attention can either be distributed over the 

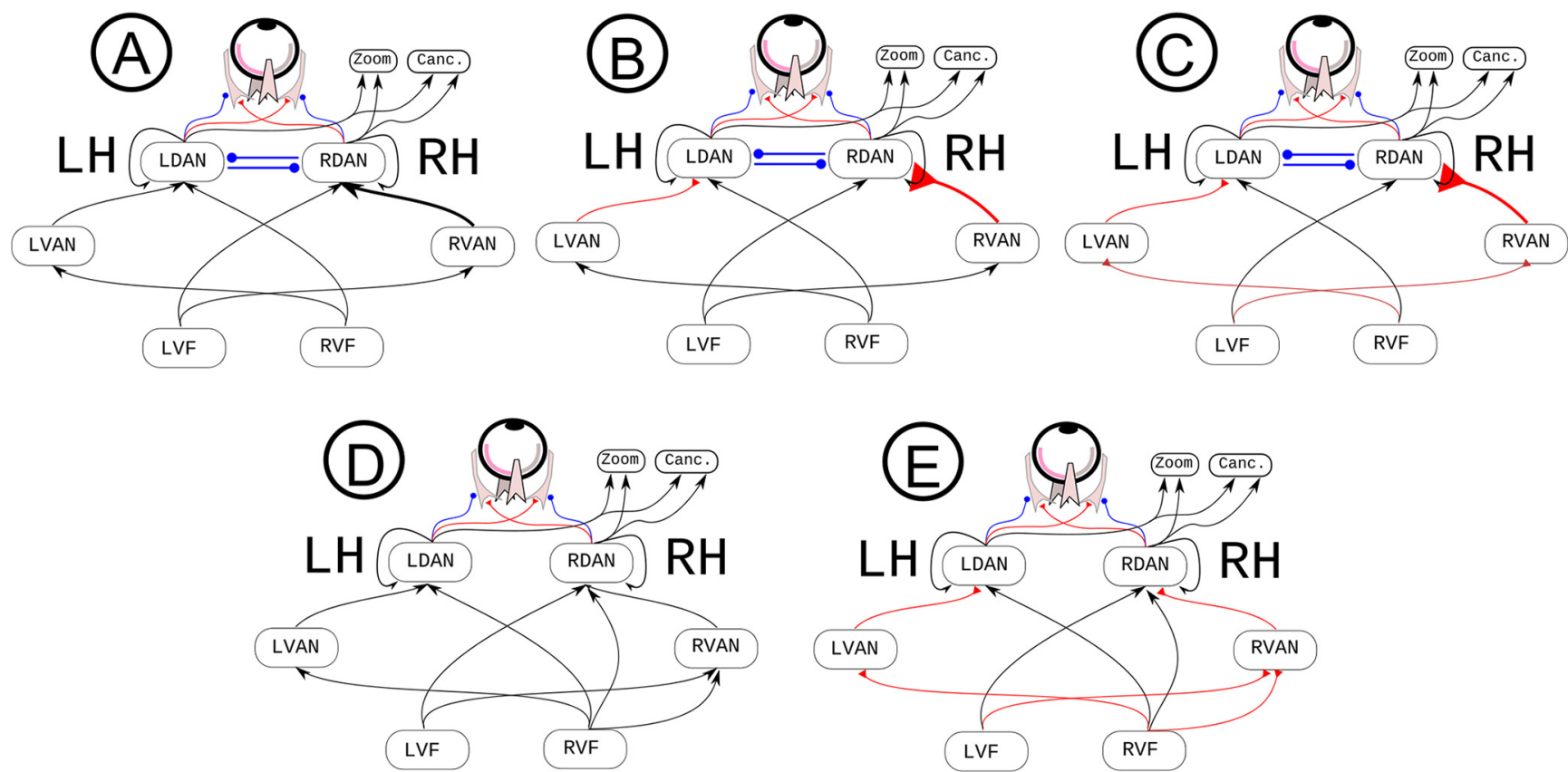

Figure 2. $\boldsymbol{A}-\boldsymbol{C}$, Different implementations of the attentional networks with interhemispheric inhibition (Koch et al., 2011) and DAN/VAN architecture (Corbetta and Shulman, 2002). D, E, Two implementations of right-hemisphere networks with bilateral competence (Heilman and Van Den Abell, 1980; Mesulam, 1981) and no interhemispheric inhibition. Arrows indicate connections that can be either excitatory or inhibitory; red connections with triangular arrowheads denote excitatory connections; blue round arrowheads represent inhibitory connections. $\mathrm{LH}$, left hemisphere; $\mathrm{RH}$, right hemisphere; Canc., cancellation units; LDAN and RDAN, dorsal attention networks in the left and in the right hemisphere, respectively; LVAN and RVAN, ventral attention networks in the left and in the right hemisphere; LVF and RVF, left and right visual field. Right and left VANs have the same number of neurons but different patterns of connection strength.

whole field, but with low resolving power, or be continuously constricted to small portions of the visual field with a concomitant increase in processing power (Eriksen and Yeh, 1985).

The artificial eye is equipped with a retina made up of a $7 \times 7$ grid of light receptors (Fig. 1). Each receptor outputs an activation value computed by averaging the luminance of the perceived stimuli across the receptive field, with radius set to 80 pixels. Receptors are evenly distributed within the artificial retina, which has a square form with a side varying from 1120 pixels (no zoom) to 96 pixels (maximum zoom). Thus, each stimulus can occupy a retinal surface ranging from $0.8 \%$ to $100 \%$ of the artificial retina. Horizontal and vertical movements of the eye are controlled by four simulated muscles (Massera et al., 2014; Fig. 1), in analogy to the medial, lateral, inferior, and superior recti of the human eye.

\section{Neural network}

We used a standard neural network model in which each node of the network has a sigmoid activation function $\varphi(x)=1 /\left(1+e^{-x}\right)$ and an adjustable threshold $\vartheta$. The output, $O$, is computed for each node $i$ by using the following equation:

$$
O_{i}=\varphi\left(A_{i}\right)
$$

Where:

$$
A_{i}=\vartheta_{i}+\sum_{j} w_{i j} O_{j}
$$

$\mathrm{w}_{i j}$ is the synaptic weight connecting unit $j$ with unit $i$. The pattern of connections between nodes has been chosen according to biological evidence on DAN and VAN in human brains (see below, Valence of VAN-DAN connections and of inter-DAN connections).

Figure $2 \mathrm{~A}$ depicts the general template network. The $7 \times 7$ retina, consisting of 49 artificial neurons, constituted the input layer. The output layer controlled the zoom with two artificial neurons, the extraocular muscles with four neurons, and a decision unit for target detection, which triggered the touch response when exceeding a criterion threshold of 0.7 . The hidden layer contained the attention networks and a hidden network devoted to control vertical eye movements (four neurons, not depicted in Fig. 1). We modeled the DAN and the VAN by building a neural model organized across two hemispheres, with visual information from each visual field projecting to the contralateral hemisphere. Each DAN had five artificial neurons; each VAN had four artificial neurons. These parameters were based on pilot work, and reflect a tradeoff between network complexity and the time needed to run simulations. With these parameters, each simulation required about a week to be completed on our hardware. The VAN-DAN connections in the right hemisphere outnumbered those in the left hemisphere, to simulate anal- 
ogous results for the human SLF II (Thiebaut de Schotten et al., 2011).

The interhemispheric connections were also modeled by following anatomic and functional results obtained in the human brain, and outlined in the Introduction. Thus, (1) they connected only the DANs, but not the VANs, which thus worked in relative isolation from one another (Catani and Thiebaut de Schotten, 2012; their Fig. 9.4D) and (2) they were inhibitory, such that each DAN inhibited the contralateral one (Koch et al., 2011): each DAN induced contralaterally-directed eye movements and inhibited ipsilaterally-directed eye movements. The DANs controlled zooming and cancellation behaviors. All the hidden units within the DANs also had reentrant connections, which integrate the previous input with the current one, thus simulating a sort of simplified visual memory, in analogy to similar mechanisms occurring in the primate brain (Salazar et al., 2012). Thus, reentrant connections resulted in some persistence of the previous inputs across steps within a given trial.

Given the importance of eye position in visually-guided target reaching (Lewis et al., 1998), we provided eye position information to neurorobots through an efference copy of the motor output. In particular, motor outputs controlling the four ocular muscles were connected one to one with the four input neurons, with a fixed weight of 1 (i.e., perfect copy from input to output).

\section{Cancellation task}

Similar to the human experiment (see Experiment 1), neurorobots performed a 30 -trial cancellation task. The human and robotic tasks were designed with the explicit constraint of being as similar as possible. Targets were presented on a virtual display measuring $512 \times 512$ pixels. At the start of each trial, the gaze of the artificial eye was initialized at the center of the display, with no zoom. Again similarly to the human experiment, each trial consisted of a set of 5 round targets, with a luminance value of 0.5 (in conventional units ranging from 0 to 1.0) and a radius of 40 pixels, randomly scattered in the virtual display. On cancellation, targets increased their luminance to the maximum value of 1.0 .

\section{The adaptive/learning process}

For the present work, neurorobots were trained by means of a genetic algorithm, a form of evolutionary computation that implements a Darwinian process of adaptation that can model cognitive development and trialand-error learning, especially when only distal rewards are available (Nolfi and Floreano, 2000; Di Ferdinando et al., 2007). Genetic algorithms are a useful alternative to supervised learning in settings such as the present one, because we employed a fitness function based on the number of cancelled targets, and not a set of input-output pairings which could be used to minimize the error by a supervised learning mechanism such as back-propagation. A typical experiment starts with the generation of a random set of individual neurorobots (each defined by a specific set of parameters of a neurocontroller). Each individual is then evaluated according to a fitness function representing the desired performance on a requested task. Due to genetic operators, such as mutation and crossover, the best individuals will populate the next generation. The process iterates until a specific performance or a fixed number of generations is reached. In the present work, each genetic string encodes the value of synaptic connections $\mathrm{w}_{i j}$ and neuron thresholds in the range $(-5$ to 5). Initially, for each evolutionary experiment a set of 100 random individuals (i.e., competing sets of parameters for the neural network of the neurorobot) were generated and evaluated for their ability to find targets. Targets had to be found as fast as possible on each of 24 cancellation trials, lasting 700 timesteps each. At the end of the evaluation phase, individuals were ranked according to their performance, and the best 20 were used to populate the next generation after having undergone a mutation process. Each parameter was encoded by an 8-bit string, thus mutations were implemented by bits switching with probability $p=0.01$. The number of generations was set to 3000 .

Three behavioral components contributed to the overall fitness, F: an exploration component, a component proportional to the number of target correctly cancelled, and a reward for responses promptness.

The exploration component, which was introduced to avoid the bootstrap problem (Nolfi and Floreano, 2000), rewarded the ability of the neurorobot to explore its visual field. In particular, the area that can be explored through eye movements was split in 100 cells. Exploration fitness $(E F)$ was then computed for each trial by dividing the number of visited cells by 100 . A second fitness component (TF) was represented for each trial by the number of correctly cancelled targets divided by 5 (i.e., the total number of presented targets). Finally, a reward for promptness $(P F)$ was given when all the five targets were cancelled. $P F$ was inversely proportional to the number of timesteps $n t$, used to cancel all the stimuli:

$P F=n t / 700$

The overall fitness was calculated as

$F=E F+T F+P F$.

After training, neurorobots' performance in the cancellation task was evaluated on 30 new trials, to measure their accuracy in finding the targets and the position of the first cancelled target, as estimated by the average value of the $X$ coordinate of the first cancelled stimulus across trials.

\section{Valence of VAN-DAN connections and of inter-DAN connections}

A set of five populations of neurorobots, each composed of 40 individuals, featured neurocontrollers with different connectional constraints. Neurocontrollers A, B, and $C$ (Fig. 2) had left-right asymmetric connections between VAN and DAN (i.e., the simulated SLF II), with a greater number of connections in the right hemisphere (120) than in the left hemisphere (108). The ratio of this asymmetry difference $(0.05)$ corresponds to the average asymmetry ratio of SLF II in 20 human subjects, as described by Thiebaut de Schotten et al. (2011; their Supplementary Table 1). In neurocontroller A (Fig. 2A), there were no constraints in terms of type of connections (inhibitory or excitatory) along the VAN and DAN. In neuro- 


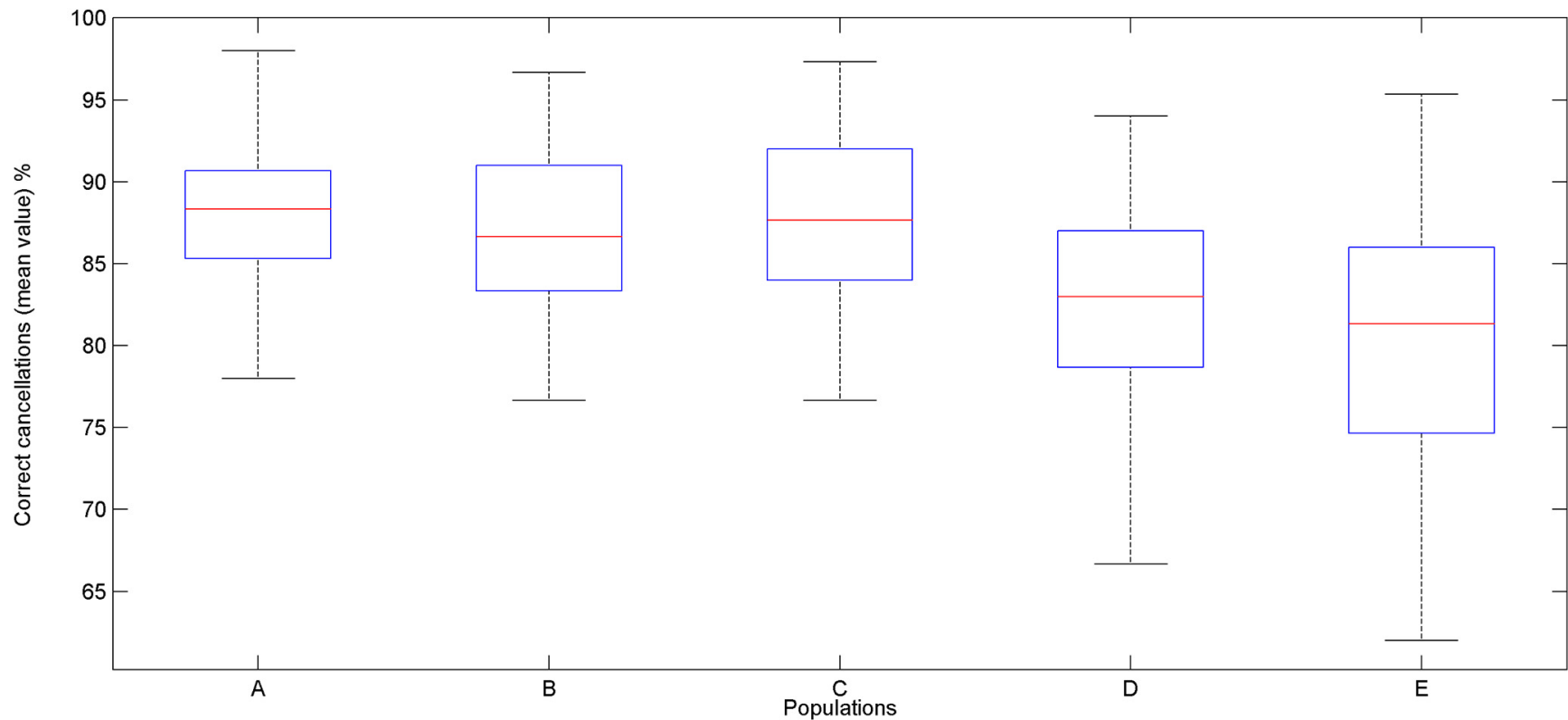

Figure 3. Mean percentage of correct cancellations computed across 30 trials for each population of 40 neurorobots provided with neurocontrollers A-E. The middle bar of the boxplot indicates the median of the tested population. The top and the bottom of the box indicate respectively the first (q1) and the third (q3) quartiles. Whisker length extends until the last data point that is not considered as an outlier, i.e., a point that is greater than $q 3+1.5 \times(q 3-q 1)$ or less than $q 1-1.5 \times(q 3-q 1)$. There were no outliers in the current dataset.

controller $\mathrm{B}$, a further constraint was added: VAN to DAN pathways were set to be excitatory during the training process (Fig. 2B). Finally, in neurocontroller $\mathrm{C}$ also the connections projecting from the retina to the VAN were set to be excitatory (Fig. 2C). To better evaluate the effect on performance of SLF II asymmetry, we trained two additional control populations based on neurocontroller C: CO with completely symmetrical VAN-DAN connections (laterality ratio $=0$ ) and C1 with VAN-DAN connections only present in the right hemisphere and absent VAN-DAN connections in the left hemisphere (complete right lateralization of SLF II).

Earlier models of spatial attention (Heilman and Van Den Abell, 1980; Mesulam, 1981) postulated a bilateral competence of the right hemisphere for both hemispaces, without explicit consideration of interhemispheric interactions. To simulate these models, we trained two additional populations of neurorobots (neurocontrollers $D$ and $E$ in Fig. 2; 40 individuals for each population). In these neurocontrollers, the right hemisphere received visual information from both the right and the left visual hemifields, while the left hemisphere received information only from the right, contralateral visual hemifield. Moreover, there were no inhibitory connections between the right DAN and its left homolog. The rest of the architecture was the same as for all the other neurocontrollers. The only difference between neurocontroller $D$ and neurocontroller $E$ was the valence of the connections running from the visual fields to VAN and DAN. In neurocontroller D, the valence of the visuo-attentional connections was not constrained and could thus assume either a positive or a negative valence. In neurocontroller $\mathrm{E}$, visuo-attentional connections were constrained to be excitatory, similar to neurocontroller $\mathrm{C}$.

Two additional control simulations were designed to assess the importance of the inhibitory valence of interDAN connections. In these simulations, we used neurorobots identical to model $\mathrm{C}$, except that the inter-DAN connections were (1) let free to evolve as excitatory or inhibitory (neurocontroller F), or (2) constrained to be facilitatory (neurocontroller G).

\section{Results}

\section{Behavioral results}

Figure 3 shows the ability of the five populations of neurobots to correctly solve the task. The mean percentages of correct cancellations are reported for each population. Figure 4 reports the performance of the populations equipped with neurocontrollers $A-E$ on correct cancellations. Each boxplot contains data collected for 40 neurorobots tested on 30 cancellation trials.

There were significant differences in the amount of correct cancellations across the populations A-E [Kruskal-Wallis test, $\left.\chi^{2}(4, n=200)=38.96, p=7.10 \mathrm{e}-08\right]$. Neurocontrollers with interhemispheric inhibition $(A-C)$ performed better than neurocontrollers without interhemispheric inhibition ( $\mathrm{D}$ and $\mathrm{E}$; post hoc pairwise comparisons using Dunn's test, all $p<0.05$ ).

Importantly, the spatial position of the first canceled target ( $X$ coordinate value for each trial; Fig. 4) did differ across the populations A-E, $\chi^{2}(4, n=200)=34.198, p=$ $4.65 \mathrm{e}-07$. The position of the first canceled target was not different from 0 (central position) in neurorobots equipped with neurocontroller $\mathrm{A}$ (Wilcoxon-Mann-Whitney, $p=0.1$, two-tailed) and with neurocontroller $\mathrm{D}(p=0.5)$. Neuroro- 


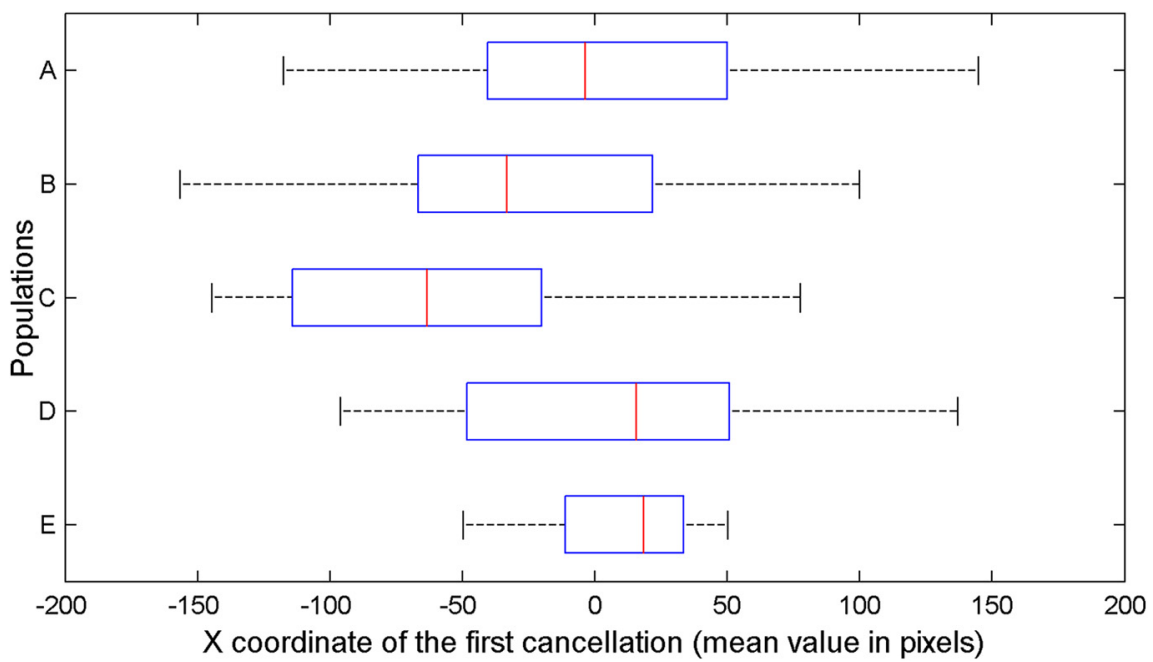

Figure 4. Average $x$ values of the first cancelled target, computed across 30 trials for each population of 40 neurorobots provided with neurocontrollers A-E.

bots $\mathrm{E}$, with bilateral competence in the right hemisphere and excitatory visual-attentional connections, showed a rightward bias, opposite to human pseudoneglect (median $=58.81, z=-2.88, p=0.004)$. Neurorobots $B$ and $\mathrm{C}$ tended instead to start their exploration from a leftsided target (neurocontroller $\mathrm{B}$, median $=-33.27, \mathrm{z}=$ $-2.06, p=0.02$; neurocontroller $\mathrm{C}$, median $=63.29, \mathrm{z}=$ $-5.35, p<0.001)$, thus showing a leftward bias reminiscent of human pseudoneglect. The control populations with complete SLF II symmetry (C0), or extreme rightward SLF II asymmetry (C1), showed the predicted patterns of performance: no pseudoneglect for C0 (median $=20.44$, $\mathrm{z}=-0.82, p=0.411)$, and large pseudoneglect for $\mathrm{C} 1$ (median $=-96.53, \mathrm{z}=-7.41, p=1.299 \mathrm{e}-13$; Fig. 5).

The additional control populations $F$ (unconstrained inter-DAN connections) and G (excitatory inter-DAN connections) achieved an overall worse performance as compared with neurorobots $C$ [Kruskal-Wallis test, $\chi^{2}(2, n=119)=$ 49.67, $p=1.635 \mathrm{e}-11$ ]. However, neurorobots $F$ (median correct cancellations, 83.33\%; 1st quartile, 79.33\%; 3rd quartile, $88.00 \%$ ) performed better than neurorobots $\mathrm{G}$ (median correct cancellations, $75.33 \%$; 1st quartile, 70.33\%; 3rd quartile, 79.67\%; Dunn's test, all $p<0.05$ ). There were also differences between populations $\mathrm{C}, \mathrm{F}$, and $G$ in the initial spatial bias [Kruskal-Wallis test, $\chi_{(2, n=119)}^{2}=$ 9.24, $p=0.0099]$. Interestingly, in population $\mathrm{F}$ inter-DAN connections had a strong tendency to evolve toward inhibition; at the end of the evolutionary process, only two of 40 individuals $(5 \%)$ had evolved excitatory connections. Perhaps as a consequence, neurorobots $\mathrm{F}$ tended to start their exploration from the left side (median $X$ value for the 1st canceled target, -77.94 pixels; 1st quartile, -119.76; 3rd quartile, -39.20 ), similar to neurorobots $C$. In contrast, neurorobots $\mathrm{G}$, with excitatory inter-DAN connections, did not show any consistent lateral bias (median $X$ value for the 1st target, -2.92 pixels; 1st quartile, -84.53; 3rd quartile, 61.95; Wilcoxon-Mann-Whitney, $p=$ 0.45 , two-tailed). These results strongly suggest that in

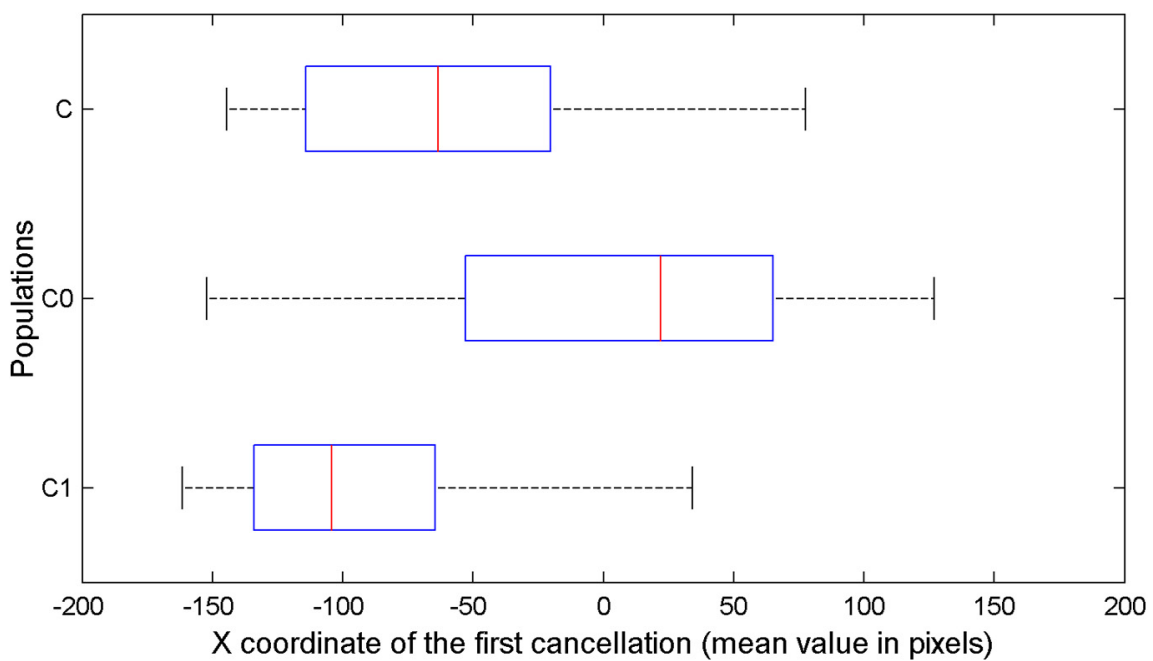

Figure 5. Average $x$ values of the first cancelled targets, for all the neurorobots provided with neurocontrollers $\mathrm{C}$, C0, and $\mathrm{C} 1$. Only average $x$ values of neurocontrollers $C$ and $C 1$ significantly differed from 0 . 


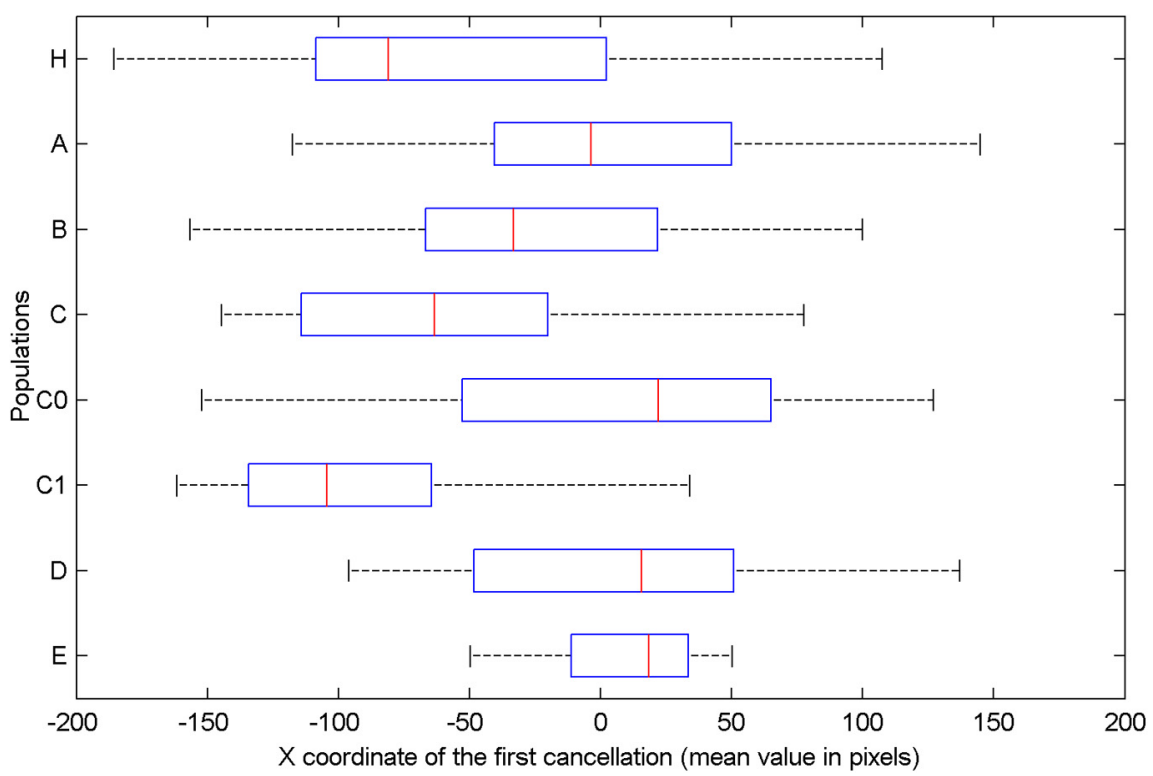

Figure 6. Average position on the $x$-axis of the first cancelled targets for human participants $(\mathrm{H})$ and for artificial neurorobots equipped with neural networks A, B, C, C0, C1, D, and E.

our setting inhibitory inter-DAN connections (1) conferred an evolutionary advantage in terms of cancellation accuracy and (2) were important to the development of pseudoneglect behavior.

\section{Neural results}

To better understand the neural dynamics leading to the exploratory bias, we examined the average activations of the DANs across all the individuals for each population, equipped with neurocontrollers $C$ (biologically-inspired asymmetry) and $\mathrm{C}_{0}$ (symmetrical attention networks). We then computed a laterality index of DAN average activations between the two hemispheres: (mean right DAN activation - mean left DAN activation)/(mean right DAN activation + mean left DAN activation), with a possible range from -1 (prevalent left DAN activity) to +1 (prevalent right DAN activity). Figure 7-10 reports the course of the laterality index across timesteps. As expected, left and right DAN activations were balanced with neurocontroller $\mathrm{C}_{0}$. On the other hand, in neurocontroller $\mathrm{C}$ activations were unbalanced toward the right hemisphere DAN. A crucial aspect for pseudoneglect concerns the initial timesteps in which the exploratory bias occurs. A higher imbalance toward the right hemisphere DAN is present at the outset of the cancellation task for neurorobots $C$, as a consequence of asymmetries in their network architecture, while it is obviously absent for neurorobots $\mathrm{C}_{0}$, with symmetrical networks. The initial imbalance favoring the right hemisphere DAN is the likely basis of the spatial bias

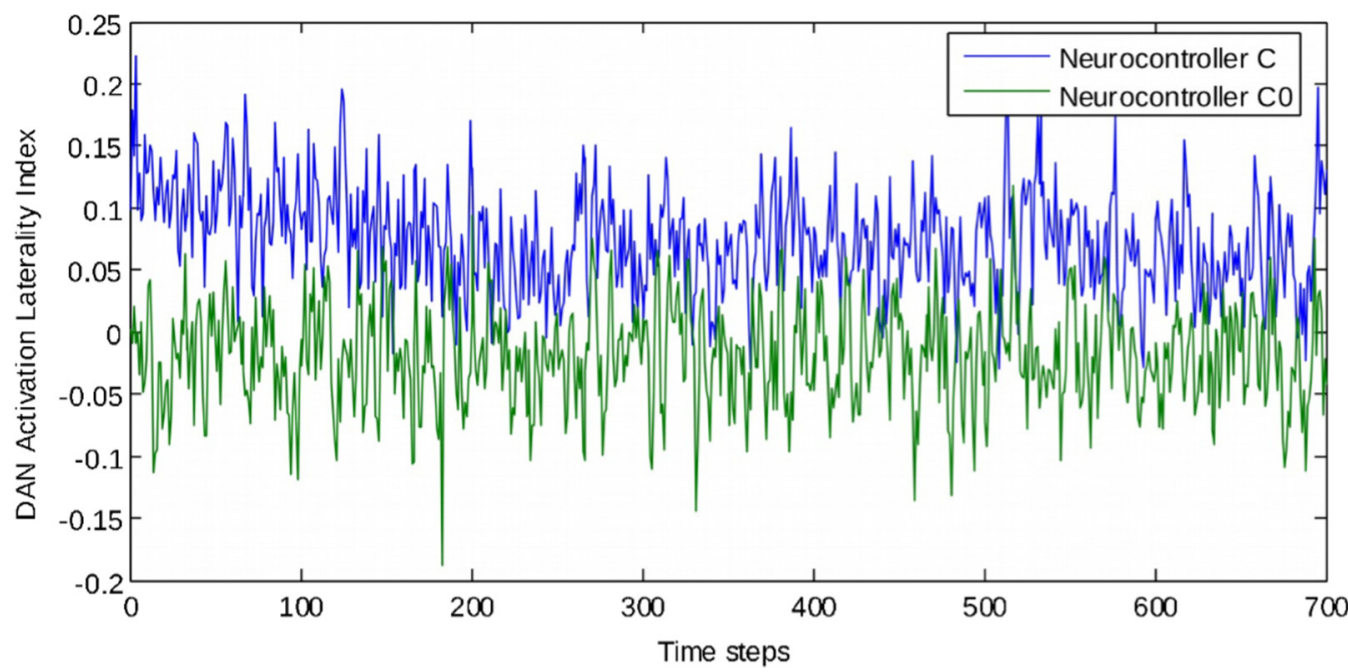

Figure 7. Laterality indexes of DAN activation computed for individuals equipped with neurocontroller $\mathrm{C}$ and $\mathrm{CO}$. A value of 0 means that activation in left and right hemisphere DANs is balanced; positive values denote prevalence of right hemisphere DAN, negative values indicate prevalence of left hemisphere DAN. 


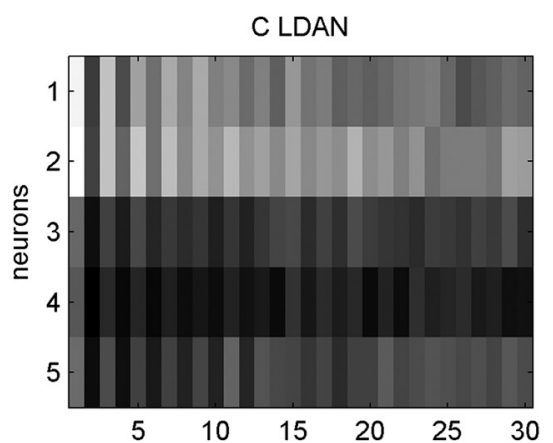

CO LDAN

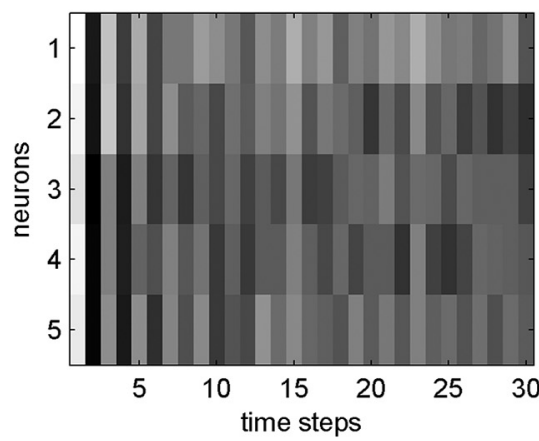

C RDAN

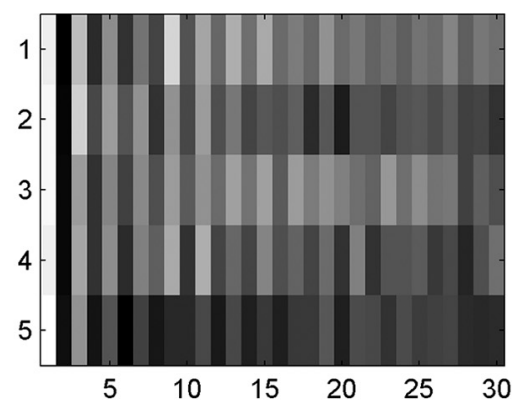

CO RDAN

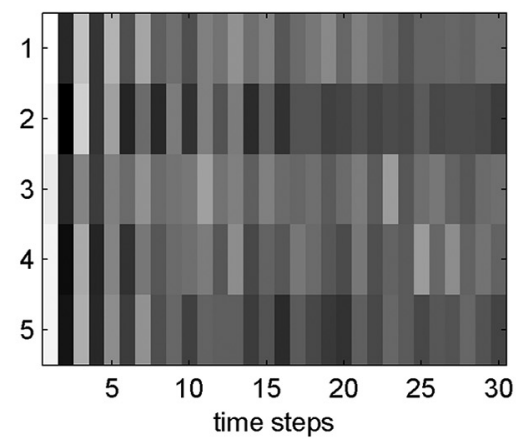

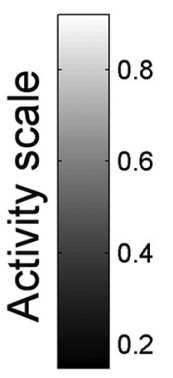

0.2

Figure 8. Average activation of hidden neurons in right hemisphere DAN (RDAN) and in left hemisphere DAN (LDAN), for the first 30 steps of individuals equipped with neurocontrollers C and C0. The activity scale goes from 0 (black) to 1 (white). Note the early, large left-right asymmetry in neurobiologically inspired $\mathrm{C}$ agents, which subsequently decreases. The symmetrical $\mathrm{CO}$ agents do not show any asymmetries of performance.
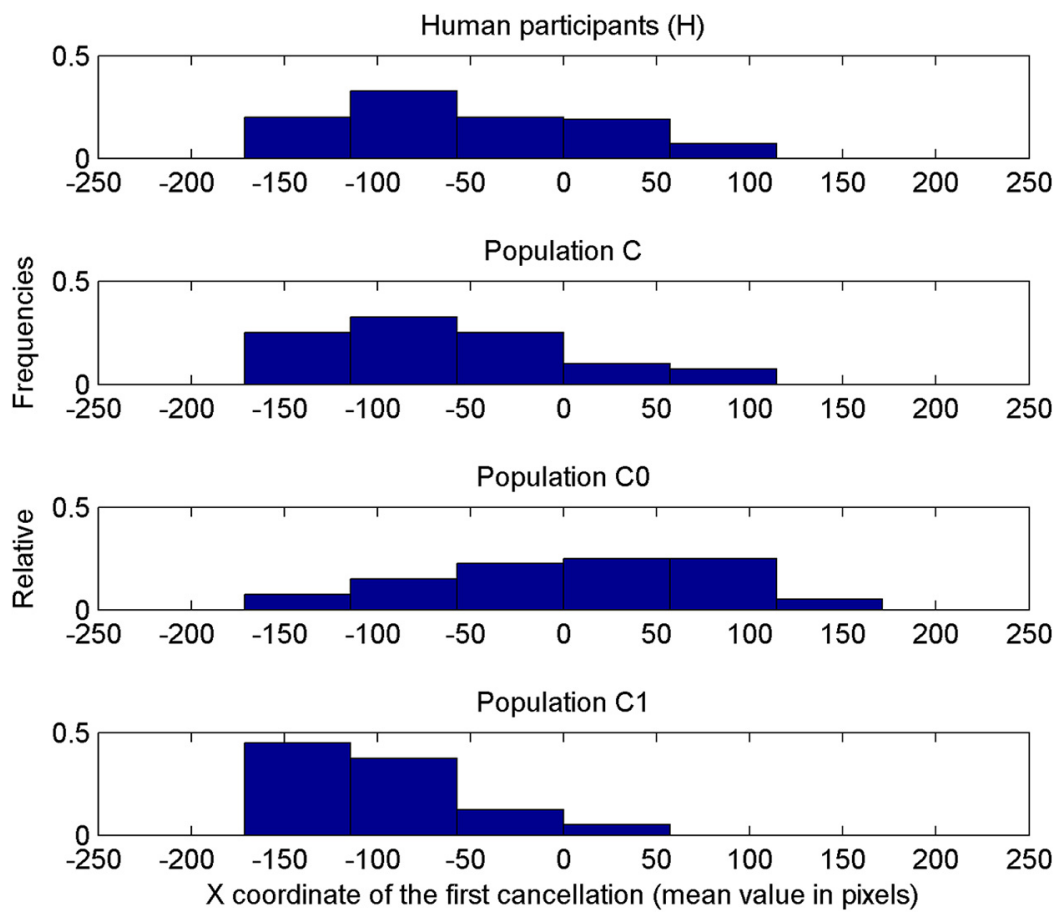

Figure 9. Relative frequencies of the distribution of the position of the first cancelled target for 101 human participants (see experiment 1) and for the populations of neurorobots $C$ (equipped with the biologically inspired neurocontroller), $C 0$ (presenting symmetrical DANs), and C1 (with VAN-DAN connections only present in the right hemisphere). 


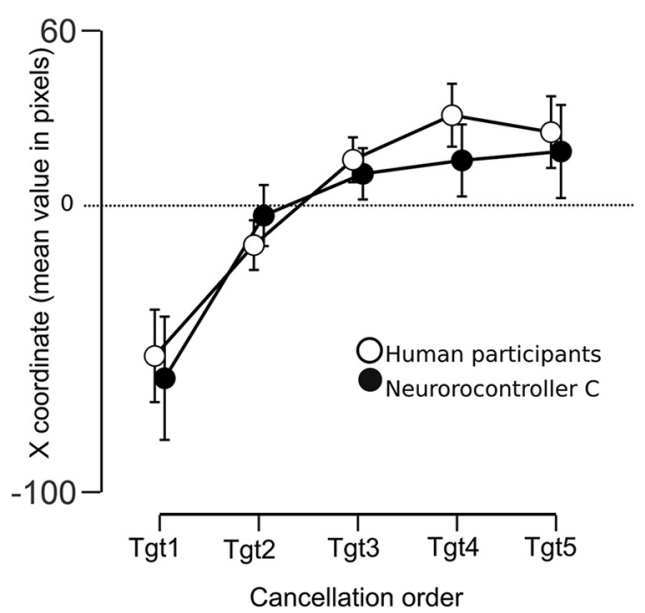

Figure 10. Coordinates of canceled targets as a function of the temporal sequence of cancellation in human participants and in neurorobot population $\mathrm{C}$. Error bars represent credible intervals of $95 \%$.

toward the initial cancellation of a left-sided item in neurorobots $\mathrm{C}$.

Figure 7-10 shows the average activation of the hidden DAN neurons in the left and in the right hemisphere during the first 30 timesteps of the cancellation task, for agents equipped with the biologically inspired neurocontroller $\mathrm{C}$, and for those equipped with the symmetrical neurocontroller $\mathrm{C}_{0}$. The initial activation is symmetrical for the $\mathrm{C}_{0}$ agents, but it is higher in the right hemisphere than in the left hemisphere for the $C$ agents. Thus, an asymmetry of VAN connections results in a corresponding activation asymmetry in the anatomically symmetrical DANs. The DAN asymmetry in the initial phases of the task is the simulated neural correlate of behavioral pseudoneglect. After the initial phase, the left-right differences are absorbed by the increased activity of the hidden units; when left and right activities reach saturation, the behavioral asymmetry decreases (see Fig. 7, where asymmetry of performance decreases around timestep 150 for neurocontroller C).

\section{Comparison between human and robotic performance}

Human participants and robotic populations as a whole did not show the same distribution of the position of the first cancelled targets [Kruskal-Wallis test, $\chi_{(5, n=301)}^{2}=$ $67.88, p<0.001$; Fig. 6]. Post hoc tests (Dunn's test with Bonferroni correction) demonstrated a difference in distribution between humans and neurocontrollers $A(p<$ $0.001)$, B $(p=0.0394), C_{0}(p<0.001)$, and $C_{1}(p=$ $0.0153)$. However, the position distribution derived from human performance and neurocontroller C's performance showed a similar degree of leftward asymmetry (Fig. 9; Dunn's test, $p=1.0$; Levene's test of homogeneity, $p=$ 0.39 ). Thus, all robotics agents performed differently from humans, with the notable exception of the neurorobot population $\mathrm{C}$, whose performance provided a good approximation to human performance.

We then compared the performance over time of human participants and model $\mathrm{C}$ neurorobots not only for the first canceled target (Figure 7-10) but also across all the remaining targets. We performed a Bayesian repeated measures ANOVA (JASP software, version 0.8.2), with agents (human, neurorobots $C$ ) as between-group factor, and the spatial position ( $\mathrm{X}$ coordinate) of the sequence of all the five canceled targets as within-group factors. The Inclusion Bayes Factor, which compares ANOVA models that contain a given effect to equivalent models stripped of the effect, showed decisive evidence (BFInclusion = $2.137 e+42$ ) for the cancellation order main effect. Thus, the order of cancellation of all the five targets depended on their spatial position (Figure 7-10). Importantly, this effect was statistically equivalent for the human and the neurorobot $\mathrm{C}$ populations. In particular, there was substantial evidence against the existence of a group main effect (BFInclusion $=0.144)$, and strong evidence against the existence of a group $X$ cancellation-order interaction $(B F I n c l u s i o n=0.046)$. These results show that the neurorobots from population $\mathrm{C}$ and human subjects behave similarly over time when canceling all the five targets.

\section{Discussion}

In this study, we established specific connectivity constraints leading to a lateral spatial bias (pseudoneglect) in artificial organisms trained to perform a visual search task by using genetic algorithms. A form of pseudoneglect that was qualitatively and quantitatively similar to that shown by normal participants did emerge in artificial neurorobots, but only in those harboring hemispheric asymmetries of connectivity that simulated those typically occurring in the human brain. As a further condition, a general excitatory influence of VAN on the ipsilateral DAN was necessary for pseudoneglect to occur in neurorobots. This novel result suggests that hemispheric asymmetry alone is not sufficient to generate a leftward bias, and thus further specifies the likely connectional constraints of pseudoneglect.

We first consider our results in the light of neurophysiological studies of pseudoneglect, and then in relation to existing modeling studies of the human attentional system. A particular instance of pseudoneglect occurs with the landmark task: When judging lines prebisected to the left of their true center, normal participants consider the left segment as being longer than the right one (Milner et al., 1992). Spatial attention has been shown to be a major determinant of this phenomenon (Toba et al., 2011). Szczepanski et al. (2010) and Szczepanski and Kastner (2013) tested normal participants' spatial bias on covert attention tasks and on the landmark task by using a multimodal approach, combining psychophysics, fMRI and transcranial magnetic stimulation (TMS). They tested only frontal and parietal ROls in the DAN, and did not explore the VAN. Their subjects' sample showed a mixed spatial bias: some subjects had a leftward bias (pseudoneglect), but most subjects showed a rightward bias (Szczepanski and Kastner, 2013). On average, the bias was rightward, unlike most of the literature results. The lateralization of the bias correlated with the lateralization index of the fMRI activation in the ensemble of the DAN ROls during a covert spatial attention task. Specifically, subjects that had more left hemisphere activation 
also had a contralateral, i.e., rightward, bias in the landmark task; conversely, subjects with more right hemisphere activation tended to have a leftward behavioral bias. TMS-induced interference on the left- or righthemisphere parietal nodes during the landmark task caused an ipsilateral shift of the bias: right parietal TMS caused a rightward shift compared to the initial bias, and left parietal stimulation caused a leftward shift. Stimulating both right and left parietal ROls did not cause a shift. Szczepanski and Kastner (2013) suggested that there is an interhemispheric competition between the DAN nodes, and the lateralization of the sum of the weights in the DAN activation shifts the attentional focus contralaterally. The possibility of long-range suppression, which might involve the DANs in both hemispheres, was shown in the monkey LIP: firing rate was suppressed when a saccade target was as far as $50^{\circ}$ from the neuron receptive field (Falkner et al., 2013).

Thus, these results are broadly consistent with the functioning of the present neurorobot population C. In agreement with Szczepanski and Kastner (2013)'s conclusions, the DAN in the current model is conceptualized as a whole, and not as separated nodes. Additionally, Szczepanski and Kastner's data showed that there is large variability between participants in the direction and degree of lateralization of DAN activation, that on average did not significantly differ between the hemispheres. Here, we aimed to explore the typical functional architecture in the human population. Therefore, we chose to model the DAN as laterally symmetrical and the VAN as right lateralized. However, there are several differences between the current models and Szczepanski et al. (2010). First, they used a landmark task while here we used a search task. Second, the overall behavioral pattern here was of a leftward classical pseudoneglect bias and not the rightward bias found by Szczepanski et al. (2010), This might result from substantial differences in the studied samples or in the tasks used. Third, and more importantly, the VAN, which has a major contribution in the current model, was not tested in their studies.

The architecture of neurorobot $C$ is partly inspired by the results of Koch et al. (2011), which might oversimplify the nature of interhemispheric interactions. Several fMRI studies of human attention areas found evidence of bilateral activation of attention areas, with a contralateral bias (Patel et al., 2015). In neurorobots D and E, we introduced bilateral competence in the right hemisphere networks (Heilman and Van Den Abell, 1980; Mesulam, 1981). However, performance in these models showed no consistent spatial bias. This suggests that right hemisphere bilateral competence by itself might not be crucial to the emergence of pseudoneglect. Moreover, in our setting the inhibitory valence of inter-DAN connections was important for the development of an initial leftwards spatial bias, as well as to reach optimal levels of performance, as stressed by additional control simulations in which interDAN connections were either set free to evolve as inhibitory or excitatory (neurorobots F), or constrained to assume only excitatory valence (neurorobots $\mathrm{G}$ ). On the other hand, evidence from neglect patients (Bartolomeo and Chokron, 1999) challenges models of attention exclusively based on interhemispheric rivalry (Kinsbourne, 1970, 1977, 1993). In addition, bilateral competence in attentional areas might be important in long-term compensation of neglect (Lunven et al., 2015; Bartolomeo and Thiebaut de Schotten, 2016). Our results stressing the importance for pseudoneglect of both right-hemisphere bilateral competence and interhemispheric competition may thus pave the way for an integrated interpretation of different lines of research on normal or dysfunctional human attention networks.

In their recent review, Borji and Itti (2013) provided a taxonomy of nearly 65 computational models of visual attention. Many of these models focused on reproducing eye movements (e.g., the saliency-based models reported in Borji and Itti, 2013), following a bottom up approach. Typically, these models extract a set of features, represented as maps, from an incoming image. Then, feature maps are combined in a saliency map where a winner-take-all mechanism will designate the spatial region to be attended. Saliency-based attention models in general do not account for exploration biases, with the exception of a recent model (Borji and Tanner, 2016), where an object center bias (the tendency to focus on the center of objects) is reproduced by adding an ad-hoc bias map to the saliency map. While important for building predictive models, this result seems of little relevance to lateral biases such as pseudoneglect. Other models (Deco and Rolls, 2004; Deco and Zihl, 2004) simulated attention as emerging from the competition of several brain areas subjected to bottom-up and top-down biases. These models do not drive eye movements; the scan path is simulated as a sequence of activations of the simulated posterior parietal cortex. Lanyon and Denham (2004, 2010) added to these models simulated eye movements and an adjustable attention window scaled according to stimuli density. Despite being successful at reproducing scan paths in healthy individuals and neglect patients, these models do not address the issue of pseudoneglect. Other models of attention did not consider pseudoneglect because of their training procedure or design constraints (Pouget and Sejnowski, 2001; Mozer, 2002; Monaghan and Shillcock, 2004; Di Ferdinando et al., 2007). Di Ferdinando et al. (2005) explored line bisection and target cancellation performance in four biologically inspired neural networks. The networks' patterns of connectivity varied along different degrees of asymmetry, inspired by specific theories. Pseudoneglect occurred in line bisection but not in visual search. In these models, motor outputs were only used for target selection; there was no active exploration of the environment, whereas when our neurorobots explored their environment the corresponding input information changed as a function of eye movements. Nonetheless, the present study shares with Di Ferdinando et al. (2005) and other work from the Zorzi group (Casarotti et al., 2012) the stress on accounts of attentional phenomena relying on sensory-motor transformations, as stated by the premotor theory of attention (Rizzolatti et al., 1987). Specifically, our results support the hypothesis that the way in which the movements of 
the actuators are controlled affects the performance on a cancellation task (Gigliotta et al., 2015).

Thus, contrary to most available models of attention, our artificial robots are trained to correctly cancel target stimuli, and are free to self-organize to find a proper solution, within the sole limits of the imposed connectivity constraints. These constraints were inspired by available data concerning the anatomic and functional organization of the attentional networks in the human brain. To the best of our knowledge, this is the first attempt to simulate pseudoneglect as a consequence of activity in the dorsal and ventral attention networks in the two hemispheres of the human brain. While this article was under review, two theoretical papers were published that also took into account the dorsal/ventral architecture of the attentional networks (Parr and Friston, 2017; Seidel Malkinson and Bartolomeo, 2017), but neither endeavored to simulate pseudoneglect. Another original feature of the present models is the embodiment factor, consisting of the explicit modeling of eye movements (Bartolomeo et al., 2002; Lanyon and Denham, 2004; Di Ferdinando et al., 2007; Miglino et al., 2009; Gigliotta et al., 2015). In particular, the present models extended the models devised by Di Ferdinando et al. (2007), by increasing the complexity of the organisms' retina, the biological plausibility of the motor system and that of the neural controllers. Conti et al. (2016) also adopted an embodied perspective, based on a humanoid robot platform. In their study, an iCub robot was trained to remove objects from a table, a task reminiscent of a cancellation task. Intrahemispheric disconnections were able to produce neglect-like behavior. However, the embodiment of the model was limited by the facts that selection of a visual target was conducted independently of the motor behavior and that the robot's eyes were kept fixed during the cancellation task. Moreover, although hemisphere asymmetry was modeled by increasing the number of right hemisphere processing units, no bias in normal performance is reported.

Moreover, contrary to most published work, our model attempted to simulate the relationships between the visual pathways and the attentional networks by respecting important biological constraints. Visual pathways project mainly to the hemisphere contralateral to each visual field. However, theoretical models of visual attention posit that the left hemisphere mainly deals with the contralateral hemispace, whereas the right hemisphere has a more bilateral competence (Heilman and Van Den Abell, 1980; Mesulam, 1981). In previous computational models this asymmetry has not always been simulated in a biologically plausible way. In some cases, both simulated hemispheres received visual information from the whole visual field, with attention asymmetries being represented in inner layers (Monaghan and Shillcock, 2004; Di Ferdinando et al., 2007). In Conti et al. (2016)'s model, the right hemisphere received information from both visual hemifields, whereas the left hemisphere processed only the contralateral visual hemifield. Our models $D$ and $E$ had similar architecture, but were unable to mimic human performance. Moreover, there is no anatomic evidence of such asymmetries in the visual pathways, and information exchange in the occipital visual areas is mainly limited to the vertical meridian (Berlucchi, 2014). In our model, these important biological constraints of visual information processing were respected, because each artificial hemisphere received visual information from the contralateral hemifield; interhemispheric connections were only present at a later stage of processing, between the artificial DANs.

It might be argued that in our model $\mathrm{C}$ a leftward bias was simply transferred or amplified from the input to the output layers. If so, however, we would have expected to observe a constant leftward bias, akin to right-sided neglect. What we found, instead, was just an initial leftward bias, at the onset of the exploration task, analogous to human physiologic pseudoneglect. To observe this initial bias, the VAN-DAN connections had to have an excitatory valence. This occurrence does not result from existing empirical data and is thus a novel prediction of the model. Also, neurorobot populations $D$ and $E$, which also had more right hemisphere than left hemisphere resources, and should then have a similar input-to-output amplification, did not show pseudoneglect, presumably because of the lack of interhemispheric inhibition.

The level of detail of the models is not a trivial matter, because it has to provide meaningful novel information while remaining tractable. A potential limitation of our study is the use of simplified versions of the frontoparietal cortical networks, without taking into consideration the substructures of the DAN and VAN, which are both broad and partly heterogeneous networks (Colby and Goldberg, 1999), nor subcortical structures such as striatum, thalamus and superior colliculus (Krauzlis et al., 2017). For example, the connectional anatomy of VAN components such as the temporoparietal junction (e.g., with the ventral cortical visual stream) and of the ventrolateral prefrontal cortex (e.g., with limbic structures) is likely to be crucial to the functioning of the VAN. Yet, our simplified model, with a VAN receiving visual input and sending excitatory connections to the ipsilateral DAN, was able to mimic human performance to an impressive level of accuracy.

More generally, our modeling is consistent with evidence from healthy subjects and neglect patients, stressing the importance of entire fronto-parietal networks, or of their dysfunction, in behavioral patterns such as pseudoneglect (Szczepanski and Kastner, 2013), or visual neglect (Corbetta and Shulman, 2011; Bartolomeo et al., 2012), respectively. Also, integrated fronto-parietal activity, with subtle, task-dependent differences in network dynamics, occurs during attention orienting in monkeys (Buschman and Miller, 2007). Concerning visual neglect, evidence suggests that a major determinant of this condition is indeed a dysfunction of the right hemisphere VAN (Corbetta and Shulman, 2011; Urbanski et al., 2011), or of its connections with the ipsilateral DAN (Thiebaut de Schotten et al., 2005).

Finally, we note that the present population-based model can be potentially used to explore in a natural manner the universal properties (the basic brain architecture) and individual differences in network efficiency, two 
aspects recently underlined by Posner (2014) as appropriate features for future models of attention.

In conclusion, we have demonstrated the emergence of pseudoneglect behavior in artificially evolving neurorobots searching for visual objects, under specific connectional constraints. These neurorobots provide a plausible model for the dynamic interactions between frontoparietal attention networks in the human brain.

\section{References}

Azouvi P, Bartolomeo P, Beis J-M, Perennou D, Pradat-Diehl P, Rousseaux M (2006) A battery of tests for the quantitative assessment of unilateral neglect. Restor Neurol Neurosci 24:273-285.

Bartolomeo P, Chokron S (1999) Left unilateral neglect or right hyperattention? Neurology 53:2023-2027. Medline

Bartolomeo P, D'Erme P, Gainotti G (1994) The relationship between visuospatial and representational neglect. Neurology 44:17101714. Medline

Bartolomeo P, Pagliarini L, Parisi D (2002) Emergence of orienting behavior in ecological neural networks. Neural Process Lett 15: 69-76. CrossRef

Bartolomeo P, Thiebaut de Schotten M, Chica AB (2012) Brain networks of visuospatial attention and their disruption in visual neglect. Front Hum Neurosci 6:110. CrossRef

Bartolomeo P, Thiebaut de Schotten M (2016) Let thy left brain know what thy right brain doeth: inter-hemispheric compensation of functional deficits after brain damage. Neuropsychologia 93:407412. CrossRef

Berlucchi G (2014) Visual interhemispheric communication and callosal connections of the occipital lobes. Cortex 56:1-13. CrossRef

Borji A, Itti L (2013) State-of-the-art in visual attention modeling. IEEE Trans Pattern Anal Mach Intell 35:185-207. CrossRef

Borji A, Tanner J (2016) Reconciling saliency and object center-bias hypotheses in explaining free-viewing fixations. IEEE Trans Neural Netw Learn Syst 27:1214-1226. CrossRef

Bowers D, Heilman KM (1980) Pseudoneglect: effects of hemispace on a tactile line bisection task. Neuropsychologia 18:491-498. Medline

Buschman TJ, Miller EK (2007) Top-down versus bottom-up control of attention in the prefrontal and posterior parietal cortices. Science 315:1860-1862. CrossRef

Casarotti M, Lisi M, Umiltà C, Zorzi M (2012) Paying attention through eye movements: a computational investigation of the premotor theory of spatial attention. J Cogn Neurosci 24:15191531. CrossRef

Catani M, Thiebaut de Schotten M (2012) Atlas of the human brain connections. Oxford: Oxford University Press.

Colby CL, Goldberg ME (1999) Space and attention in parietal cortex. Annu Rev Neurosci 22:319-349. CrossRef Medline

Conti D, Di Nuovo S, Cangelosi A, Di Nuovo A (2016) Lateral specialization in unilateral spatial neglect: a cognitive robotics model. Cogn Process 17:321-328. CrossRef Medline

Corbetta M, Shulman GL (2002) Control of goal-directed and stimulus-driven attention in the brain. Nat Rev Neurosci 3:201215. CrossRef

Corbetta M, Shulman GL (2011) Spatial neglect and attention networks. Annu Rev Neurosci 34:569-599. CrossRef Medline

Deco G, Rolls ET (2004) A neurodynamical cortical model of visual attention and invariant object recognition. Vis Res 44:621-642. CrossRef

Deco G, Zihl J (2004) A biased competition based neurodynamical model of visual neglect. Med Eng Phys 26:733-743. CrossRef

Di Ferdinando A, Casarotti M, Vallar G, Zorzi M (2005) Hemispheric asymmetries in the neglect syndrome: a computational study. In: Modelling language, cognition and action (Cangelosi A, Bugmann G, Borisyuk R, eds), pp 249-258. Singapore: World Scientific.
Di Ferdinando A, Parisi D, Bartolomeo P (2007) Modeling orienting behavior and its disorders with "ecological" neural networks. J Cogn Neurosci 19:1033-1049. CrossRef

Eriksen CW, Yeh YY (1985) Allocation of attention in the visual field. J Exp Psychol Hum Percept Perform 11:583-597. CrossRef

Falkner AL, Goldberg ME, Krishna BS (2013) Spatial representation and cognitive modulation of response variability in the lateral intraparietal area priority map. J Neurosci 33:16117-16130. CrossRef

Gigliotta O (2017) Simulated embodied agents for visual search. GitHub repository. Retrieved December 22, 2017. Available at https://gitlab.com/TalMal/Simulated_embodied_agents_for_visualsearch.git.

Gigliotta O, Bartolomeo P, Miglino O (2015) Neuromodelling based on evolutionary robotics: on the importance of motor control for spatial attention. Cogn Process 16 [Suppl 1]:237-240. CrossRef

Heilman KM, Van Den Abell T (1980) Right hemisphere dominance for attention: the mechanism underlying hemispheric asymmetries of inattention (neglect). Neurology 30:327-330. CrossRef

Jewell G, McCourt ME (2000) Pseudoneglect: a review and metaanalysis of performance factors in line bisection tasks. Neuropsychologia 38:93-110. Medline

Kinsbourne M (1970) A model for the mechanism of unilateral neglect of space. Trans Am Neurol Assoc 95:143-146.

Kinsbourne M (1977) Hemi-neglect and hemisphere rivalry. In: Hemiinattention and hemisphere specialization, Vol 18 (Weinstein EA, Friedland RP, eds), pp 41-49. New York: Raven Press.

Kinsbourne M (1993) Orientational bias model of unilateral neglect: evidence from attentional gradients within hemispace. In: Unilateral neglect: clinical and experimental studies (Robertson IH, Marshall JC, eds), pp 63-86. Hove: Lawrence Erlbaum Associates.

Koch G, Cercignani M, Bonnì S, Giacobbe V, Bucchi G, Versace V, Caltagirone C, Bozzali M (2011) Asymmetry of parietal interhemispheric connections in humans. J Neurosci 31:8967-8975. CrossRef Medline

Krauzlis RJ, Bogadhi AR, Herman JP, Bollimunta A (2017) Selective attention without a neocortex. Cortex. Advance online publication. Retrieved September 1, 2017. pii: S0010-9452(17)30283-6. CrossRef.

Lanyon LJ, Denham SL (2004) A model of active visual search with object-based attention guiding scan paths. Neural Netw 17:873897. CrossRef

Lanyon LJ, Denham SL (2010) Modelling visual neglect: computational insights into conscious perception. PLoS One 5:e11128. CrossRef Medline

Lewis RF, Gaymard BM, Tamargo RJ (1998) Efference copy provides the eye position information required for visually guided reaching. J Neurophysiol 80:1605-1608.

Lunven M, Thiebaut De Schotten M, Bourlon C, Duret C, Migliaccio R, Rode G, Bartolomeo P (2015) White matter lesional predictors of chronic visual neglect: a longitudinal study. Brain 138:746-760. CrossRef

Marzi CA (2010) Asymmetry of interhemispheric communication. Wiley Interdiscip Rev Cogn Sci 1:433-438. CrossRef

Massera G, Ferrauto T, Gigliotta O, Nolfi S (2014) Designing adaptive humanoid robots through the FARSA open-source framework. Adapt Behav 1059712314536909.

McCourt ME, Jewell G (1999) Visuospatial attention in line bisection: stimulus modulation of pseudoneglect. Neuropsychologia 37:843855. CrossRef

McCourt ME, Garlinghouse M, Reuter-Lorenz PA (2005) Unilateral visual cueing and asymmetric line geometry share a common attentional origin in the modulation of pseudoneglect. Cortex 41: 499-511. CrossRef

Mesulam M-M (1981) A cortical network for directed attention and unilateral neglect. Ann Neurol 10:309-325. CrossRef

Mesulam M-M (1999) Spatial attention and neglect: parietal, frontal and cingulate contributions to the mental representation and attentional targeting of salient extrapersonal events. Philos Trans R Soc Lond B Biol Sci 354:1325-1346. CrossRef 
Miglino O, Ponticorvo M, Bartolomeo P (2009) Place cognition and active perception: a study with evolved robots. Connect Sci 21:314. CrossRef

Milner AD, Brechmann M, Pagliarini L (1992) To halve and to halve not: an analysis of line bisection judgements in normal subjects. Neuropsychologia 30:515-526. CrossRef

Monaghan P, Shillcock R (2004) Hemispheric asymmetries in cognitive modeling: connectionist modeling of unilateral visual neglect. Psychol Rev 111:283-308. CrossRef

Mozer MC (2002) Frames of reference in unilateral neglect and visual perception: a computational perspective. Psychol Rev 109:156185. CrossRef

Nolfi S, Floreano D (2000) Evolutionary robotics: the biology, intelligence, and technology. Cambridge: MIT Press.

Nolfi S, Gigliotta O (2010) Evorobot. In: Evolution of communication and language in embodied agents, pp 297-302. Berlin: Springer.

Ossandón JP, Onat S, König P (2014) Spatial biases in viewing behavior. J Vis 14:20.

Parr T, Friston KJ (2017) The computational anatomy of visual neglect. Cereb Cortex. Advance online publication. Retrieved November 28, 2017. CrossRef

Patel GH, Yang D, Jamerson EC, Snyder LH, Corbetta M, Ferrera VP (2015) Functional evolution of new and expanded attention networks in humans. Proc Natl Acad Sci USA 112:9454-9459. CrossRef Medline

Posner MI (2014) Guides to the study of attention. In: The Oxford handbook of attention (Nobre AC, Kastner S, eds), pp 1-5. Oxford: Oxford University Press.

Pouget A, Sejnowski TJ (2001) Simulating a lesion in a basis function model of spatial representations: comparison with hemineglect. Psychol Rev 108:653-673. CrossRef

Rizzolatti G, Riggio L, Dascola I, Umiltá C (1987) Reorienting attention across the horizontal and vertical meridians: evidence in favor of a premotor theory of attention. Neuropsychologia 25:31-40. CrossRef

Rousseaux M, Beis JM, Pradat-Diehl $\mathrm{P}$, Martin $\mathrm{Y}$, Bartolomeo $\mathrm{P}$, Bernati T, Chokron S, Leclercq M, Louis-Dreyfus A, Marchal F, Perennou D, Prairial C, Rode G, Samuel C, Sieroff E, Wiart L,
Azouvi $P$ (2001) [Presenting a battery for assessing spatial neglect. Norms and effects of age, educational level, sex, hand and laterality]. Rev Neurol (Paris) 157:1385-1401.

Salazar R, Dotson N, Bressler S, Gray C (2012) Content-specific fronto-parietal synchronization during visual working memory. Science 338:1097-1100. CrossRef

Schenkenberg T, Bradford DC, Ajax ET (1980) Line bisection and unilateral visual neglect in patients with neurologic impairment. Neurology 30:509-517. Medline

Seidel Malkinson T, Bartolomeo P (2017) Fronto-parietal organization for response times in inhibition of return: the FORTIOR model. Cortex. Advance online publication. Retrieved November 21, 2017. pii: S0010-9452(17)30378-7. CrossRef

Szczepanski SM, Kastner S (2013) Shifting attentional priorities: control of spatial attention through hemispheric competition. $J$ Neurosci 33:5411-5421. CrossRef

Szczepanski SM, Konen CS, Kastner S (2010) Mechanisms of spatial attention control in frontal and parietal cortex. J Neurosci 30:148160. CrossRef Medline

Thiebaut de Schotten M, Urbanski M, Duffau H, Volle E, Lévy R, Dubois B, Bartolomeo P (2005) Direct evidence for a parietalfrontal pathway subserving spatial awareness in humans. Science 309:2226-2228. CrossRef

Thiebaut de Schotten M, Dell'Acqua F, Forkel SJ, Simmons A, Vergani F, Murphy DGM, Catani M (2011) A lateralized brain network for visuospatial attention. Nat Neurosci 14:1245-1246. CrossRef

Toba MN, Cavanagh P, Bartolomeo P (2011) Attention biases the perceived midpoint of horizontal lines. Neuropsychologia 49:238246. CrossRef Medline

Urbanski M, Bartolomeo P (2008) Line bisection in left neglect: the importance of starting right. Cortex 44:782-793. CrossRef Medline

Urbanski M, Thiebaut de Schotten M, Rodrigo S, Oppenheim C, Touzé E, Méder JF, Moreau K, Loeper-Jeny C, Dubois B, Bartolomeo P (2011) DTI-MR tractography of white matter damage in stroke patients with neglect. Exp Brain Res 208:491-505. CrossRef 